\title{
A New Composition for Co(II)-porphyrin-based Membranes Used in Thiocyanate-selective Electrodes
}

\author{
Dana Vlascici ${ }^{1}$, Eugenia Fagadar-Cosma ${ }^{2, *}$ and Otilia Bizerea-Spiridon ${ }^{1}$ \\ ${ }^{1}$ West University of Timisoara, Faculty of Chemistry-Biology-Geography, Inorganic Chemistry \\ Department, 16 Pestalozzi Str., Timisoara-300115 (Romania) \\ 2 Institute of Chemistry Timisoara of Romanian Academy, 24 M. Viteazul Ave., Timisoara-300223 \\ (Romania), Fax: +40 256491824
}

* Author to whom correspondence should be addressed. E-mail: efagadar@yahoo.com

Received: 22 April 2006 / Accepted: 26 July 2006 / Published: 24 August 2006

\begin{abstract}
In the present paper, the potentiometric response characteristics of a metalloporphyrin-based electrode in o-nitrophenyloctylether (o-NPOE) plasticized polyvinyl chloride (PVC) membrane are presented for a set of monovalent anions. As membrane ionophore, 5,10,15,20-tetrakis-(4-methoxyphenyl)-porphyrin-Co(II) (CoTMeOPP) was used. To establish the optimum composition of the membrane, different molar percents of cationic derivative (mol.\% relative to ionophore) were used. Electrodes formulated with membranes containing $1 \mathrm{wt} \%$ ionophore, $66 \mathrm{wt} . \%$ o-NPOE, $33 \mathrm{wt} \%$ PVC (plasticizer: PVC $=2: 1$ ) and the lipophilic cationic derivative (35 mol\%) are shown to exhibit high selectivity for thiocyanate with a near-Nernstian slope in the working concentration range of $1.0 \times 10^{-1}-1.0 \times 10^{-5} \mathrm{M}$, with a good stability in time.
\end{abstract}

Keywords: metalloporphyrins, thiocyanate, anion-selective electrode, near-Nernstian slope.

\section{Introduction}

Substituted meso-tetraarylporphyrins are an interesting class of molecular building blocks owing to their large size, extended $\pi$-system and versatility of metal ion binding.

Among a lot of other applications, metalloporphyrins are used as ionophores in solvent / polymeric membrane electrodes. They can induce potentiometric anion selectivity patterns different from the 
classical Hofmeister pattern. Porphyrins are interesting anion carriers because of their ability to have a Lewis acidic metal as the coordinating site. The binding affinity of this central metal could be controlled by the surrounding porphyrin ring as well as the fifth or sixth ligand attached to the metal. In these cases, electrode selectivity towards anions is not governed by anion lipophilicity as in the case of dissociated ion-exchanger, but by specific chemical interactions between the metalloporphyrin from the membrane and the anions in the sample solution [1-5]. It has been shown that the nature of the ionophore-anion interaction is influenced by the charge of the central metal ion from the porphyrin structure. The operative mechanism (neutral carrier versus charged carrier) must be known, because the optimization of membrane permselectivity is highly dependent on the incorporation of additional membrane additives. It has been shown that electrode response and selectivity is enhanced by the addition of tetraphenylborate derivatives when the metalloporphyrins are acting as charged carriers (which is the case of metal(IV)-porphyrins), while tetraalkylammonium species are required as additives for membrane doped with ionophores that function as neutral carriers (the case of metal(II)porphyrins) [6].

The first use of CoTMeOPP as ionophore in an anion-selective electrode was reported by Gupta et al. [7] for the determination of molybdate ions. The electrode was PVC based, using NaTPB as anion excluder and dibutylphtalate (DBP) as plasticizer, with a ratio composition of ionophore: PVC: DBP (12:60:100).

Our electrode is also consisting of a PVC membrane but we have used a different plasticizer: $o$ nitrophenyloctilether (o-NPOE) and a lipophilic cationic derivative: trioctylmethyl-ammonium chloride (TOMACl). The amount of the membrane components was also different: 1 wt.\% ionophore, 66 wt.\% o-NPOE, 33 wt.\% PVC (plasticizer: PVC = 2:1) with various concentrations of cationic additive: $12 \% \mathrm{~mol}$, 35\% mol. and 50\% mol. TOMACl (relative to the ionophore). In these conditions, the electrode shows affinity for the thiocyanate ions over a lot of other monovalent anions and the obtained results are presented below.

Thiocyanate is the major toxic metabolite of hydrogen cyanide, a toxic substance the organism may be exposed to, as a result of cigarette smoking or industrial pollution. Thiocyanate represents a byproduct of tobacco that accumulates in blood and tissues of smokers. Besides, it is also one of the major constituents of waste water from the gasification of coal factories, in which various hazardous by-products are formed.

The toxicity of thiocyanate is significantly less than that of cyanide, but chronically elevated levels of blood thiocyanate can inhibit the uptake of iodine by the thyroid gland. Serum thiocyanate has been validated as a predictor of total and coronary 15-year mortality, being either superior or equal to the smoking questionnaire in two populations, respectively $[8,9]$.

A lot of thiocyanate sensors based on different ionophores were reported for the selective determination of this compound from biological samples (saliva and urine), for example: 5,10,15,20tetratolylporhine manganese(III) chloride [5], 5,10,15,20-tetraphenylporphine rhodium(III) chloride [10] or bis-[N-(2-hydroxyethyl)salicylaldimino]copper(II) [11]. We have chosen the above mentioned thiocyanate-selective electrodes, because the selectivity coefficients were determined by separate solution method (SSM) [12], which was also used in the present study. 


\section{Results and Discussion}

The potentiometric response characteristics of the liquid / polymeric membrane electrodes based on ionophore CoTMeOPP were determined using solutions from $1.0 \times 10^{-5}$ to $1.0 \times 10^{-1} \mathrm{M}$ of the following anions: $\mathrm{ClO}_{4}^{-}$, $\mathrm{SCN}^{-}, \mathrm{I}^{-}$, Salicylate ${ }^{-} \mathrm{F}^{-}, \mathrm{Cl}^{-}, \mathrm{NO}_{2}^{-}, \mathrm{NO}_{3}^{-}$in $0.05 \mathrm{M}$ of 4-morpholino-ethanesulfonic acid (MES), adjusted to $\mathrm{pH} 5.5$ with $\mathrm{NaOH}$.

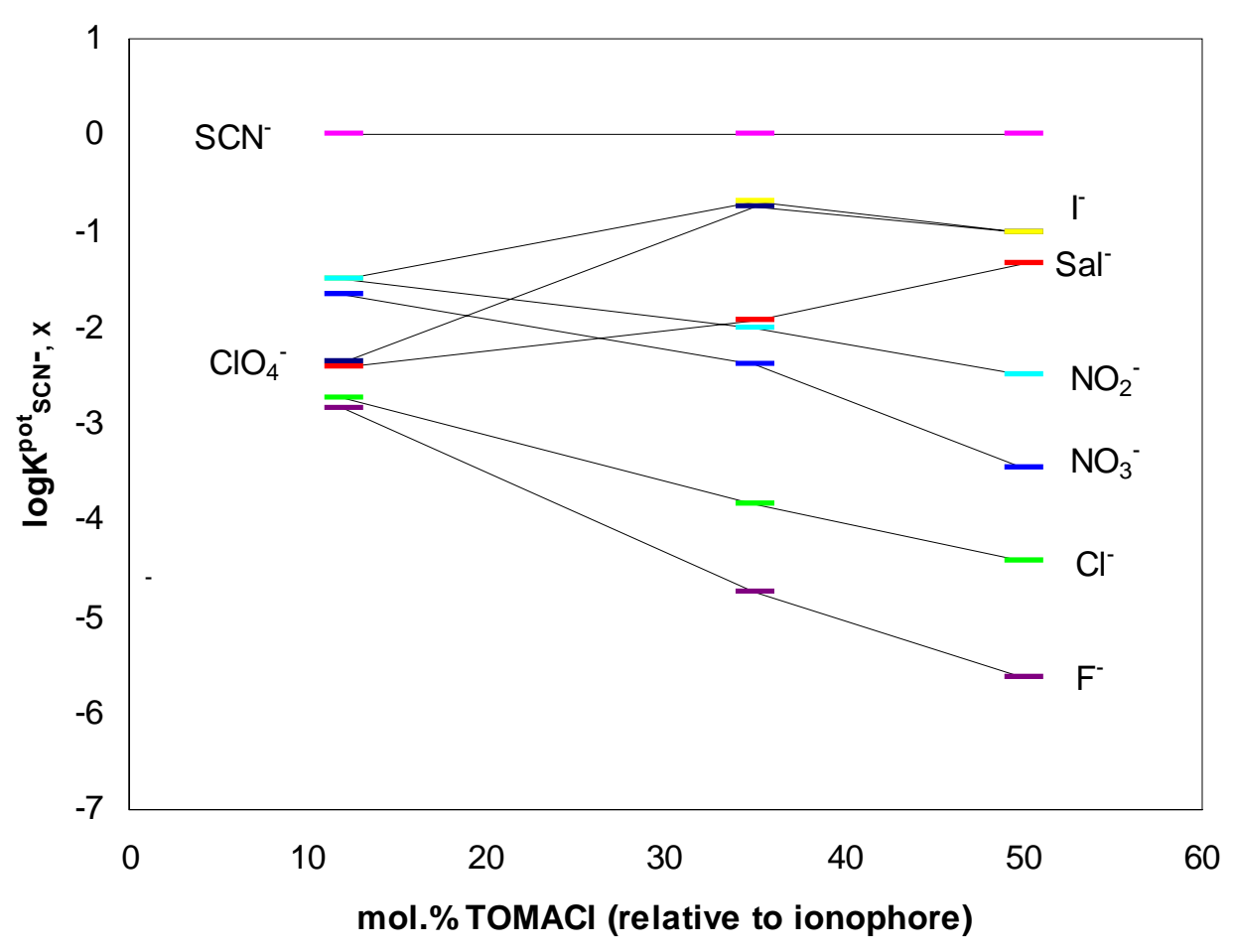

Figure 3. Selectivity coefficients, $\operatorname{logK}_{\mathrm{SCN}^{-}, \mathrm{X}^{-}}^{\text {pot }}$, for PVC/o-NPOE membranes doped with ionophore CoTMeOPP and various concentrations of cationic additive

The potentiometric response characteristic of an ion-selective electrode depends not only on the nature of the ionophore, but also significantly on the nature and amount of the additives used. To establish the optimum composition of the membrane, 3 electrodes were prepared having the following composition: 1 wt.\% ionophore CoTMeOPP, 66 wt.\% o-NPOE, 33 wt.\% PVC with various concentrations of cationic additive: $12 \% \mathrm{~mol}, 35 \% \mathrm{~mol}$. and $50 \% \mathrm{~mol}$. TOMACl (relative to the ionophore). The selectivity coefficients of these electrodes are comparatively presented in Fig. 3.

Analizing Fig. 3 it can be concluded that the incorporation of trioctylmethylammonium chloride (TOMACl) as a cationic additive in the membrane arise in an enhancement of the anionic response for thiocyanate. The three proposed electrodes performed a strong affinity to thiocyanate over all the other tested anions, developing anti-Hofmeister behavior.

The potentiometric response of the tested electrodes to thiocyanate in MES buffer 5.5 is presented in Fig. 4. 


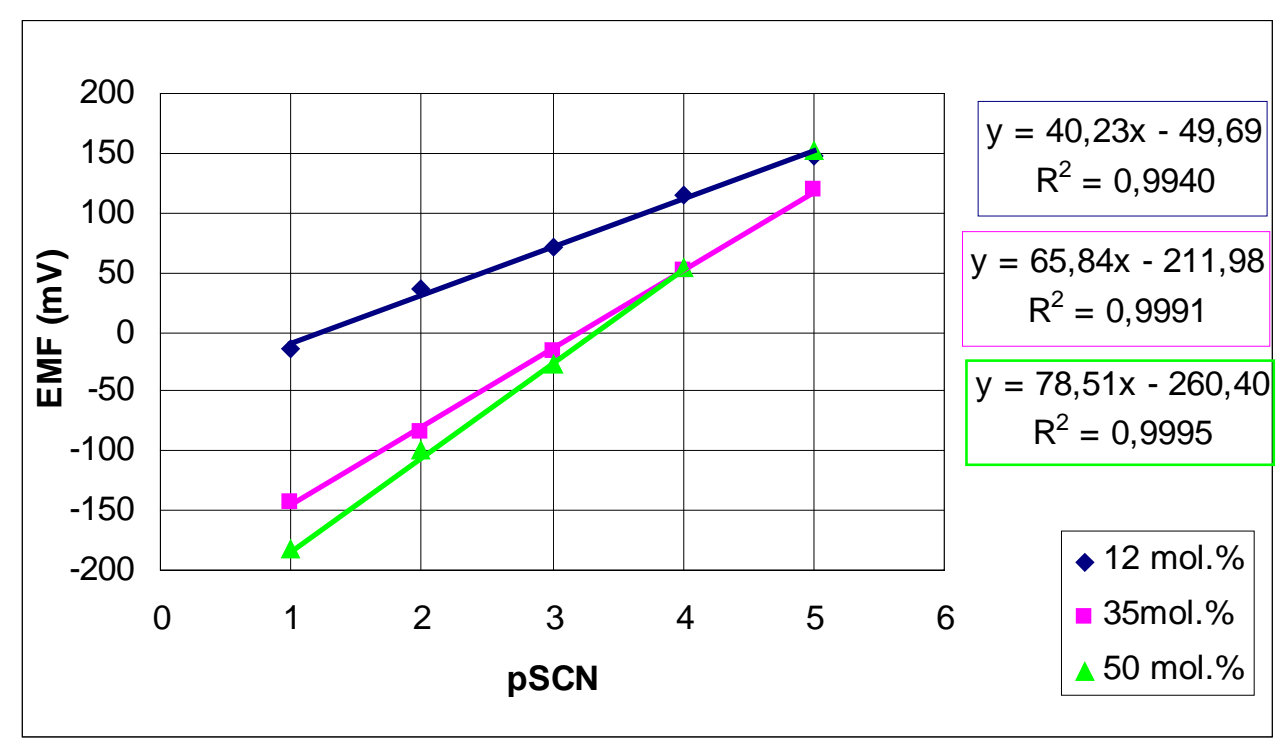

Figure 4. Potentiometric responses of membranes containing ionophore CoTMeOPP in PVC/o-NPOE matrix to thiocyanate in MES buffer, $\mathrm{pH}=5.5$ and various concentrations of cationic additive.

As it can be seen from Fig. 3 and 4, the widest linear range, the lowest limit of detection, the best values of the selectivity coefficients and a near-Nernstian slope were observed for the electrode with 35 mol\% of cationic additive (TOMACl) added. So, we can conclude that the optimal composition of the thiocyanate-selective electrode membrane is: 1 wt.\% ClCoTPP, 66 wt.\% o-NPOE, 33 wt.\% PVC (plasticizer: PVC $=2: 1$ ) and $35 \mathrm{~mol} . \%$ of TOMACl (relative to ionophore), with a slope of $-(65.8 \pm$ 1.0) $\mathrm{mV} /$ decade.

The selectivity coefficients of the electrode having the optimal membrane composition are comparatively presented with the corresponding values of the thiocyanate-selective electrodes having the ionophores 5,10,15,20-tetratolylporhine manganese(III) chloride [5], 5,10,15,20-tetraphenylporphine rhodium(III) chloride [10] or bis-[N-(2-hydroxyethyl)salicylaldimino]copper(II) [11], as can be seen in Table 1.

Table 1. Selectivity coefficients for different thiocyanate-selective electrodes (SSM).

\begin{tabular}{|l|c|c|c|c|}
\hline \multirow{2}{*}{$\begin{array}{c}\text { Interferin } \\
\text { g anion }\end{array}$} & \multicolumn{4}{|c|}{$\log \mathrm{K}_{\mathrm{SCN}^{-}, \mathrm{X}^{-}}^{\mathrm{pot}}$} \\
\cline { 2 - 5 } & Ref. [5] & Ref. [10] & Ref. [11] & This work \\
\hline $\mathrm{F}^{-}$ & $-3,44$ & $-3,80$ & - & $-4,80$ \\
$\mathrm{Sal}^{-}$ & $-1,62$ & $-1,96$ & $-2,40$ & $-1,95$ \\
$\mathrm{Cl}^{-}$ & $-3,88$ & $-3,71$ & $-3,00$ & $-3,84$ \\
$\mathrm{I}^{-}$ & $-2,49$ & $-1,39$ & $-2,30$ & $-0,85$ \\
$\mathrm{ClO}_{4}{ }^{-}$ & $-1,54$ & $-0,71$ & $-1,90$ & $-0,93$ \\
$\mathrm{NO}_{3}{ }^{-}$ & $-2,90$ & $-2,87$ & $-3,20$ & $-2,46$ \\
$\mathrm{NO}_{2}{ }^{-}$ & $-3,88$ & $-1,71$ & $-2,90$ & $-2,00$ \\
\hline
\end{tabular}

By comparing the results listed in Table 1, it can be underlined that our electrode shows similar selectivity coefficients to the thiocyanate-sensors previously prepared [5,10,11], with a good value regarding the chloride anion, which is the most important interfering anion from the biological fluids. 
The potentiometric response of the electrode with $35 \mathrm{~mol} \%$ of cationic additive (TOMACl) for various anions is presented in Fig. 5.

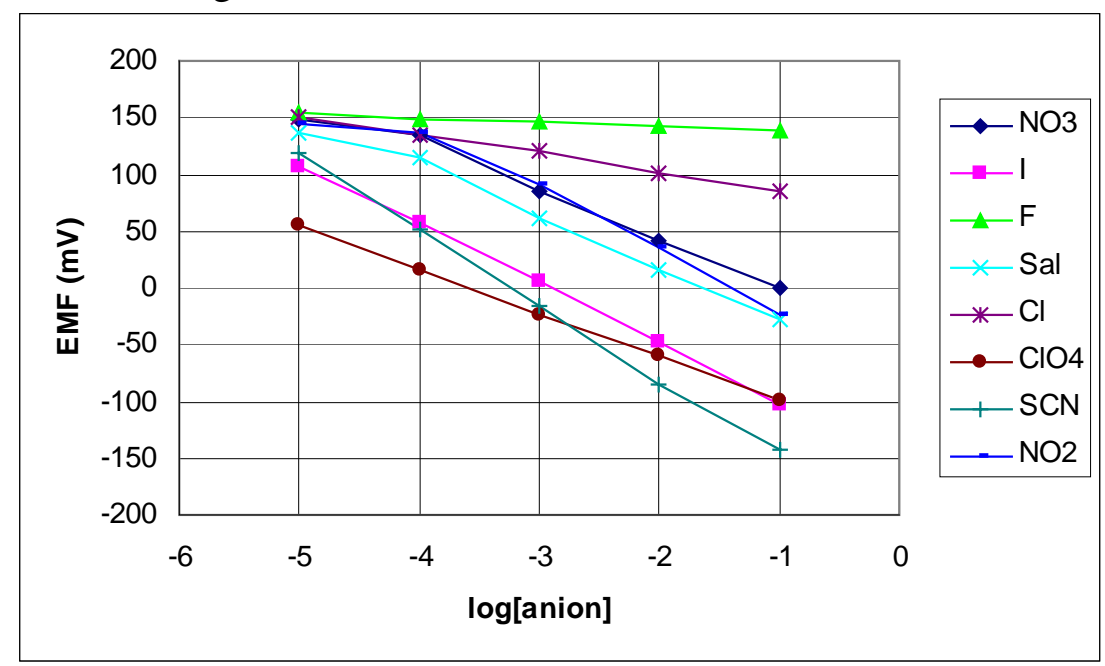

Figure 5. Potentiometric anion responses of the electrodes prepared with a membrane having the optimal composition.

For the electrodes having the optimum composition of the membrane, the response time, detection limit, lifetime and working $\mathrm{pH}$ range were determined.

The average time for the thiocyanate-selective electrode to reach the final potential value after successive immersion of the electrode in a series of thiocyanate ion solutions, each having a 10-fold difference in concentration, was measured. The obtained response time was about $20 \mathrm{~s}$ as the concentration of thiocyanate was changed from $10^{-4}$ to $10^{-3} \mathrm{M}$. For lower concentrations of the solution the response time was the same, but it became longer for concentrated solutions.

The detection limit for the best performing electrode was established at the point of intersection of the extrapolated linear mid-range and final low concentration level segments of the calibration plot and it is $6 \times 10^{-6} \mathrm{M}$.

The working $\mathrm{pH}$ range of the optimum electrode was determined by measuring the potentials in a $\mathrm{pH}$ range of 2.0 to 11.3 at $1.0 \times 10^{-2} \mathrm{M} \mathrm{SCN}^{-}$solution. The $\mathrm{pH}$ of the solutions was adjusted with $\mathrm{HCl}$ and $\mathrm{NaOH}$ and the obtained results are presented in Fig 6.

The working $\mathrm{pH}$ range of the sensor, in which the potential remains constant, is $3.0-9.5$ (Fig. 6).

In order to determine the lifetime of the thiocyanate-selective electrode, the slope of the three electrodes having the optimum composition of the membrane was measured. A little decrease of the slope in time was observed, but after six weeks of intensive use, it still remains in the analytical useful range.

\section{Analytical applications}

In human biological samples (saliva and urine), the analytical control of thiocyanate can be potentiometrically performed by using an ion-selective electrode. For the potentiometric determination of thiocyanate in urine and saliva, measurements were carried out by standard addition method, on two 
types of samples: from a cigarette smoker and from a non-smoker. The samples were diluted by a factor of 10 with MES buffer at pH 5.5 .

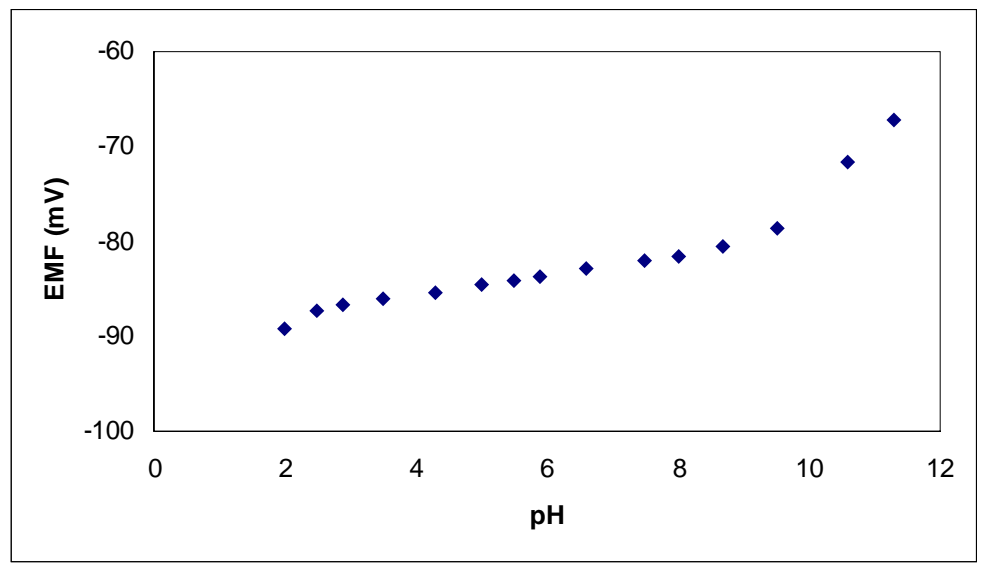

Figure 6. Effect of $\mathrm{pH}$ on cell potential.

The results were compared with a colorimetric method used as the reference method, and are presented in Table 2.

Table 2. Determination of thiocyanate in biological samples.

\begin{tabular}{|c|c|c|c|}
\hline \multirow[b]{2}{*}{ Sample } & \multirow[b]{2}{*}{ Method } & Non-smoker & Smoker \\
\hline & & \multicolumn{2}{|c|}{$\mathrm{SCN}^{-}(\mathrm{mol} / \mathrm{L}) \pm \mathrm{S}^{\mathrm{a}}$} \\
\hline \multirow[t]{2}{*}{ Urine } & Potentiometry & $(2.8 \pm 0.1) \times 10^{-4}$ & $(8.2 \pm 0.2) \times 10^{-4}$ \\
\hline & $\begin{array}{l}\text { Spectrophotometr } \\
\mathrm{y}\end{array}$ & $(2.7 \pm 0.2) \times 10^{-4}$ & $(8.3 \pm 0.1) \times 10^{-4}$ \\
\hline \multirow[t]{2}{*}{ Saliva } & Potentiometry & $(5.6 \pm 0.1) \times 10^{-4}$ & $(1.7 \pm 0.1) \times 10^{-3}$ \\
\hline & $\begin{array}{l}\text { Spectrophotometr } \\
\mathrm{y}\end{array}$ & $(5.7 \pm 0.2) \times 10^{-4}$ & $(1.8 \pm 0.1) \times 10^{-3}$ \\
\hline
\end{tabular}

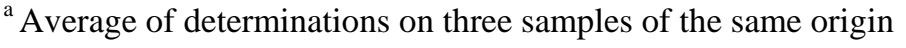

By analyzing the results presented in Table 2, a good agreement between the control colorimetric and potentiometric method is revealed.

\section{Experimental Section}

\subsection{Reagents}

5,10,15,20-Tetrakis(4-methoxyphenyl)-21H,23H-porphyrin and ionophore 5,10,15,20-tetrakis-(4methoxyphenyl)-porphyrin-Co(II) were synthesized in accordance with previously published procedures and characterized by UV-VIS, IR, ${ }^{1} \mathrm{H}-\mathrm{RMN}$ and MS spectra. For polymer membrane preparation, o-nitrophenyloctylether (o-NPOE), trioctylmethyl-ammonium chloride (TOMACl), PVC (high mol.wt.), tetrahydrofuran (THF, distilled prior to use) were purchased from Fluka and Merck. 
All aqueous solutions were prepared with salts of the highest purity available. The sample solutions for all potentiometric measurements consisted of sodium salts of the given anions in $0.05 \mathrm{M}$ 4morpholino-ethanesulfonic acid (MES), adjusted to $\mathrm{pH}=5.5$ with $\mathrm{NaOH}$.

\subsection{ISE membrane formulation and EMF measurements. Apparatus}

The composition of the PVC membrane was: $1 \mathrm{wt} \%$ CoTMeOPP, $66 \mathrm{wt} \%$ o-nitrophenyloctylether (o-NPOE), 33 wt.\% PVC (plasticizer: PVC = 2:1). Trioctylmethylammonium chloride (TOMACI) was used as additive in membrane (different \%mol. relative to the ionophore).

First, the ionophore and the additive were dissolved in the solvent mediator. Then the PVC and sufficient amount of THF were added and mixed to obtain a transparent solution. This mixture was transferred onto a glass plate of $20 \mathrm{~cm}^{2}$, and the THF was allowed to evaporate at room temperature leaving a tough flexible membrane trapped in a PVC matrix. The cast membranes were approximately $150 \mu \mathrm{m}$ thick.

Potentiometric measurements were performed with the following galvanic cell:

\section{$\mathrm{Hg} / \mathrm{Hg}_{2} \mathrm{Cl}_{2}$ /bridge electrolyte/sample/ion-selective membrane/Ag(Hg)/internal cable.}

The bridge electrolyte consisted of $0.1 \mathrm{M} \mathrm{KNO}_{3}$. Prior to EMF measurements, the electrodes were conditioned for $24 \mathrm{~h}$ in a $0.01 \mathrm{M} \mathrm{NaCl}$ solution. All experiments were performed at ambient temperature $\left(22 \pm 2^{\circ} \mathrm{C}\right)$. Potentials were measured using a Hanna Instruments HI8817 pH/mV-meter. Potentiometric selectivity coefficients were determined according to the separate solution method [12] by using the experimental EMF values obtained for $0.1 \mathrm{M}$ solutions of the test anions and a theoretical slope of $-59.2 \mathrm{mV} /$ decade for the primary anion. Activity coefficients were assumed to be constant for all analyzed anions, and no correction was made for the slight changes in the liquid junction potential of the reference electrode.

\subsection{Method of synthesis for the meso-tetra(p-methoxyphenyl)-porphyrine}

The synthesis was done by adapting the literature data [13, 14] using stoechiometric amounts of propionic anhydride in order to capture the water generated during the reaction and in this way to improve the yield(24\%).

\subsection{Method of synthesis for 5,10,15,20-tetrakis-(4-methoxyphenyl)-porphyrin-Co(II) (CoTMeOPP)}

The complex was synthesized according to literature data [7, 15] by boiling a mixture of 5,10,15,20-tetrakis(4-methoxyphenyl)porphyrin and of cobalt acetate in glacial acetic acid.

\section{Conclusion}

The results of the present study put into evidence that the electrodes having 5,10,15,20-tetrakis-(4methoxyphenyl)-porphyrin-Co(II) as ionophore in o-nitrophenyloctylether plasticized PVC membranes, realized with an internal electric solid contact, exhibit a relatively high selectivity for thiocyanate over many anions. The most important characteristics of the best performing electrodes are: slope: $-(65.8 \pm 1.0) \mathrm{mV} /$ decade; linear range: $1 \times 10^{-1}-1 \times 10^{-5} \mathrm{M}$; detection limit: $6 \times 10^{-6} \mathrm{M}$; 
response time: 20 s. The electrode having the optimal membrane composition was used for the determination of thiocyanate in urine and saliva, on two types of samples: from a cigarette smoker and from a non-smoker.

\section{References and Notes}

1. Malinowska, E.; Meyerhoff, M.E.; Role of axial ligation on potentiometric response of Co(III) tetraphenylporphyrin-doped polymeric membrane to nitrite ion, Anal. Chim. Acta 1995, 300, 3343.

2. Bakker, E.; Pretsch, E. Lipophilicity of tetraphenylborate derivatives as anionic sites in neutral carrier-based solvent polymeric membranes of corresponding ion-selective electrochemical and optical sensors, Anal. Chim. Acta 1995, 309, 7-17.

3. Chaniotakis, N.A.; Chasser, A.M.; Meyerhoff, M.E.; Groves, J.T. Influence of porphyrin structure on anion selectivities of manganese (III) porphyrin-based membrane electrodes, Anal. Chem. 1988, 60, 185-188.

4. Shamsipur, M.; Khayatian, G.; Tangestaninejad, S. Thiocyanate-selective membrane electrode based on (octabromotetraphenylporphyinato) manganese(III) chloride, Electroanalysis 1999, 11, 1340-1348.

5. Khorasani, J.H.; Amini, M.K.; Mataghi, H.; Tangestaninejad, S.; Moghadam, M. Manganese porphyrin derivatives as ionophores for thiocyanate-selective electrodes: the influence of porphyrin substituents and additives on the response properties, Sensors and Acuators B 2002, 87, 448-456.

6. Bakker, E.; Malinowska, E.; Schiller, R.D.; Meyerhoff, M.E. Anion-selective membrane electrodes based on metalloporphyrins. The influence of lipophilic anionic and cationic sites on potentiometric selectivity, Talanta 1994, 41, 881-890.

7. Gupta, Vinod K.; Chandra, S.; Chauhan, D.K.; Mangla, R. Membranes of 5,10,15,20-Tetrakis(4Methoxyphenyl) Porphyrinatocobalt (TMOPP-Co) (I) as $\mathrm{MoO}_{4}{ }^{2-}$ - Selective, Sensors 2002, 2 , 164-173.

8. Heliövaara, M. et al. Serum thiocyanate concentration and cigarette smoking in relation to overall mortality and to deaths from coronary heart disease and lung cancer, J. Chron. Dis. 1981, 34, 305311.

9. Meberg, A.;Marstein, S. Smoking During Pregnancy: Effects on the Fetal Thyroid Function, Acta Paediatrica Scandinavica 1986, 75(5), 762-766.

10. Vlascici, D.; Spiridon-Bizerea, O.; Făgădar-Cosma, E., Thiocyanate-selective electrode based on rhodium porphyrin derivates, J. Optoelectron. Adv. Mat. 2006, 8(2), 883-887

11. Huang, X.; Chai, Y.; Yuan, R.; Wang, X.; Li, Q., Highly selective thiocyanate electrode based on bis-[N-(2hydroxyethyl)salicylaldimino]copper(II) complex as a neutral carrier, Anal. Sci., 2004, 20, 1185-1188

12. Umezawa, Y.; Buhlmann, P.; Umezawa, K.; Tohda, K.; Amemiya, S. Potentiometric selectivity coefficients of ion-selective electrodes. Part I. Inorganic cations, Pure Appl. Chem. 2002, 74 (6), 923-994. 
13. Walker, F. A. An ESR Study of Coordination to the Fifth and Sixth Position of $\alpha, \beta, \gamma, \delta$-Tetra-(pmethoxyphenyl)porphinatocobalt(II). J. Am. Chem. Soc. 1970, 92, 4235-4244.

14. Adler, A.D.; Longo, F.R.; Goldmacher, J.; Assour, J.; Korsakoff, L. A simplified synthesis for meso-tetraphenylporphine. J. Org. Chem. 1967, 32, 476-487.

15. Dorough, G.D.; Miller, J.R.; Huennekens, F.M. Spectra of the Metallo-derivatives of $\alpha, \beta, \gamma, \delta$ Tetraphenylporphine, J. Am. Chem.Soc. 1951, 73, 4315-4320.

(c) 2006 by MDPI (http://www.mdpi.org). Reproduction is permitted for noncommercial purposes. 